\title{
Ubiquitous Access to Personalised Services
}

\author{
${ }^{1}$ Tore E. Jønvik, ${ }^{2}$ Anne Marie Hartvigsen $\&{ }^{3}$ Do van Thanh \\ 1. Unik - University of Oslo - Norway - tlf: +4790199176-torejoen@ifi.uio.no \\ 2. AgderUniversity College - Norway - tlf: +4795755 499-amhartvi@stud.hia.no \\ 3. Telenor R\&D - Norway - tlf: +47909 7710 2-thanh-van.do@telenor.com
}

\begin{abstract}
Nowadays, the user expects not only to be able to access services and applications anywhere at anytime and on any terminal fixed or mobile; but also in the same way as at home. In order to provide ubiquitous access to personalised services this paper propose to implement the User Profile containing the user's preferences, settings and personal data, as an XML web service which is accessible to the user at anytime anywhere and on any terminal. As an XML Web service, the User Profile will also be accessible from any application over operator's borders, firewall and without the need of a specific middleware platform, e.g. CORBA, RMI, DCOM, etc. With such a User Profile, ubiquitous and customised access to applications and services will be a reality. The paper starts with a brief overview of the XML Web service concept. The User profile is described in details before the main focus, which is about realising the User Profile as an XML service. In the conclusion, further works and activities are proposed.
\end{abstract}

Key words: XML Web services, Web technology, User profiles, Distributed Computing, Ubiquitous service access, Service customisation, Preferences and Settings

\section{INTRODUCTION}

Nowadays, the user expects not only to be able to access services and applications anywhere at anytime and on any terminal fixed or mobile; but also in the same way as at home. The functionality, the behaviour, the presentation, the look and feel, the preferences and settings, etc. should preferably be the same or as close as possible to what he/she is used to. Of course, it is always possible for a skilled user to set up and customise an

\footnotetext{
The original version of this chapter was revised: The copyright line was incorrect. This has been corrected. The Erratum to this chapter is available at DOI: 10.1007/978-0-387-35618-1_37
} 
application to his taste, and to restore the original settings after use. However, it is a boring and time consuming task that he/she would prefer to be exempt from. For a non-technical user such a task can neither be required nor accepted.

In telecommunications, the User Profile is used to capture the user's preferences, settings and personal data such as address/telephone list, bookmarks, family photos, encryption keys, etc. In order to make the User profile available to any desired application or service at the same time as to allow the user to access and modify it at anytime anywhere and on any terminal, we propose in this paper to make the User Profile available on the World Wide Web as an XML Web service.

As an XML Web service, the User Profile will be accessible from any application over operator's borders, firewall and without the need of a specific middleware platform, e.g. CORBA, RMI, DCOM, etc. With such a User Profile, ubiquitous and customised access to applications and services will be a reality. The paper starts with a brief overview of the XML Web service concept. The User profile is described in details before the main focus, which is about realising the User Profile as an XML service. In the conclusion, further works and activities are proposed.

\section{USING XML IN DISTRIBUTED COMPUTING}

Distributed Computing is not a new research area. It was addressed already by CORBA, RMI, DCOM, etc. What XML web services additionally offer is an XML based access via transparent Internet protocols like HTTP, relieving distributed computations from the need of middleware platforms and enabling the use of services across company borders, firewalls, infrastructures and technologies.

Several large software companies and standardisations organisations have gathered behind the Web service concept, putting a lot of work, money and marketing efforts into it. However, there exists a lot of confusion and divergent opinions of what Web Services are and what they should be used for.One might classify Web Services as any kinds of services that are available on the World Wide Web. Often this would include customer focused services that are available through Web browsers, such as tracking a shipment or maintaining a customer profile through a Web interface that connects to underlying systems.

In this paper, by XML Web services it is meant self-contained, modular applications that can be described, published, located, and invoked over a network [1]. Specifically these applications use XML for data description, SOAP (Simple Object Access Protocol) for messaging (invocation), WSDL 
(Web Service Description Language) for service description (in terms of data types, accepted messages, etc) and UDDI (Universal Description, Discovery and Integration) for publishing and discovery. Figure 1 shows how the protocols play together in the Web Service architecture. The idea with XML Web services is that one application should be able to dynamically exploit the functionality of other applications, which is exposed as Web services. The applications offering Web services might themselves use other Web services, either for accessing content and business logic, or to utilise supporting services such as security, billing, orchestration, etc. [2].

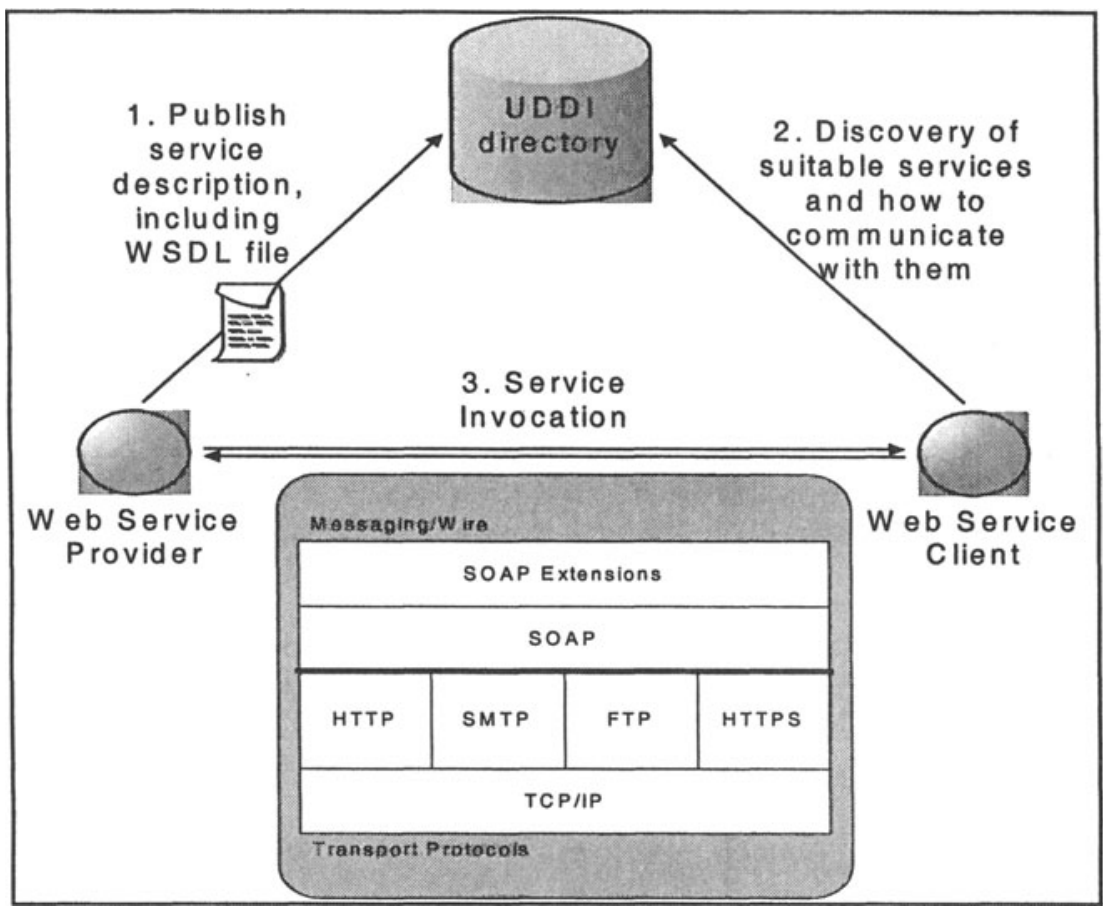

Figure 1. Web Service Protocol Architecture

\section{WHAT IS A USER PROFILE?}

In telecommunication systems, in order to allow the customisation of services, a User Profile is introduced to capture the preferences and settings of the users as in the case of UPT (Universal Personal Telecommunication) [3] and in TINA (Telecommunications Information Networking Architecture) [4]. In GSM (Global System for Mobile Communications), such a User Profile is called Subscriber Data or Subscriber Profile [5]. The 
User Profile contains information, which is required for service provisions, identification, authentication, routing, call handling, charging, subscriber tracing, operation, and maintenance purposes. Unfortunately, the User Profile as defined has many limitations. The User Profile is intended for the customisation of the main service, namely voice communication or telephony, and its supplementary services, e.g. call forwarding, call answering, etc. It is also stored deep inside the operator's system and is hardly available to the $3^{\text {rd }}$ applications or services.

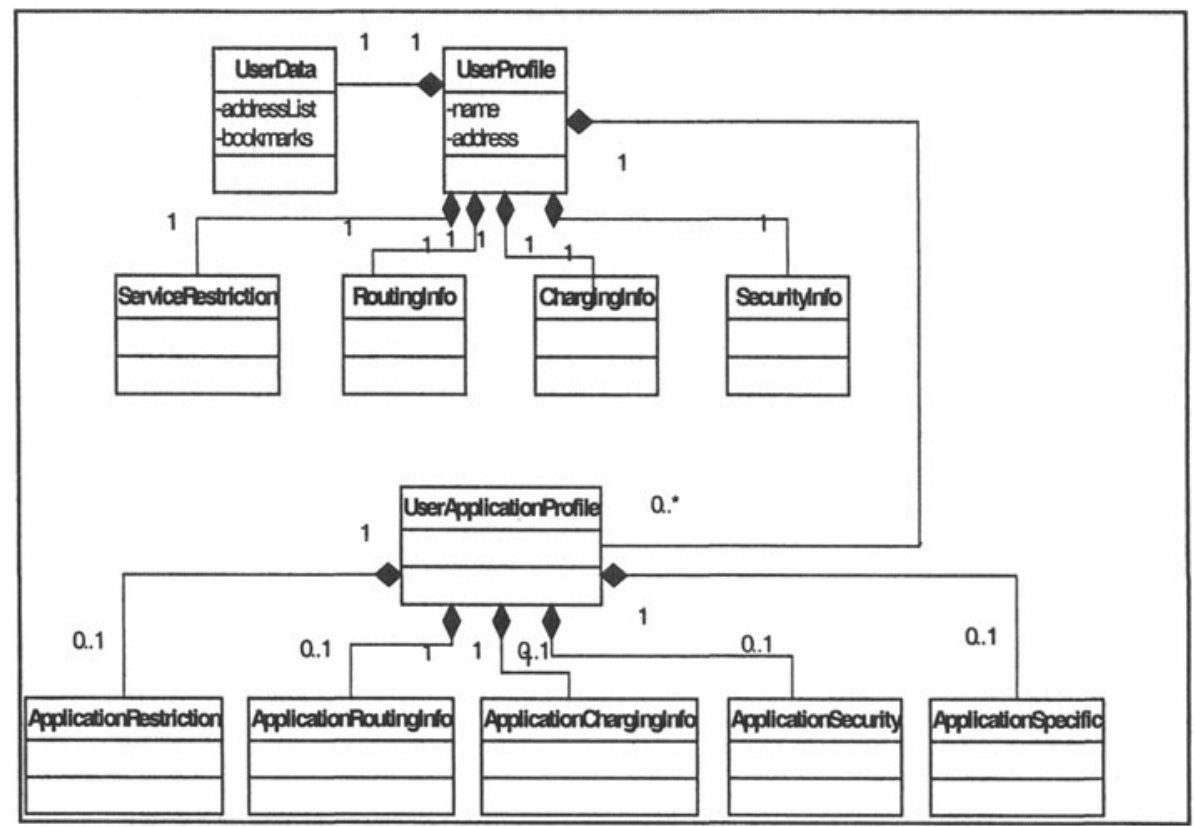

Figure 2. The User Profile structure

In order to allow the users access to multiple applications and services anytime, anywhere and on any terminal, the content of the User Profile needs to be extended to fulfil the following requirements:

- For each user the User Profile shall be expandable to incorporate the preferences and settings for any additional application or service that the user requires.

- For each application the User Profile shall contain the information necessary for the presentation of the application on the terminal types requested by the user.

- For each application the User Profile shall contain the usage restriction

- The User Profile shall incorporate also user's data such as address book, telephone list, bookmark or favourite link list, etc. 
We propose a structure for the User Profile in UML (Unified Modeling Language) in Figure 2.

\section{USER PROFILE AS AN XML WEB SERVICE}

Several issues need to be addressed in the design of the User Profile Web Service (UPWS):

- Storage and retrieval of user profile data

- User access to and modification of user profile data

- 3rd party application access to user profile data

To manage the data retrieval and providing the UPWS we need a small application, and we will call it the User Profile Manager (UPM). This application will act as a middle layer between the data itself and the user terminal or application.

\subsection{Storage and retrieval of user profile data}

When deciding how to store and manage XML data there are at least four alternative ways to do it: In a file system, in a native XML database, in a modified object database or in a relational database. File systems and native $\mathrm{XML}$ databases are appropriate when one is storing $\mathrm{XML}$ documents rather that XML data, the latter being more structured. Object databases have been identified by several observers as a natural storage for XML, but have yet to prove their usefulness in this context. When storing structured data, it is generally recommended to use a relational database and then extract the information from the database to XML when needed.

Structured data will benefit from the relational model when it comes to retrieval, searching and aggregation of data. While this forces a need to transform, or decompose the original XML document, decomposing an XML document to persist it to a relational database is not all that difficult. Also, many relational database vendors are implementing thin XML serialiser wrappers that enable them to generate XML documents on demand from relational data. So even if data will be coming in and going out as XML, e.g. in the form of Web services SOAP messages, they can be stored as relational data.

One obvious advantage with using relational databases is that the technology is mature, stable and ubiquitous, and there exists a whole range of tools for working with relational databases.

The UPM will extract the relational data into XML documents before providing applications and users access to them. It will also have to extract the data from XML documents and update the database. Depending on the 
implementation, more or less of this functionality can reside in the database itself.

\subsection{User access to and modification of user profile data}

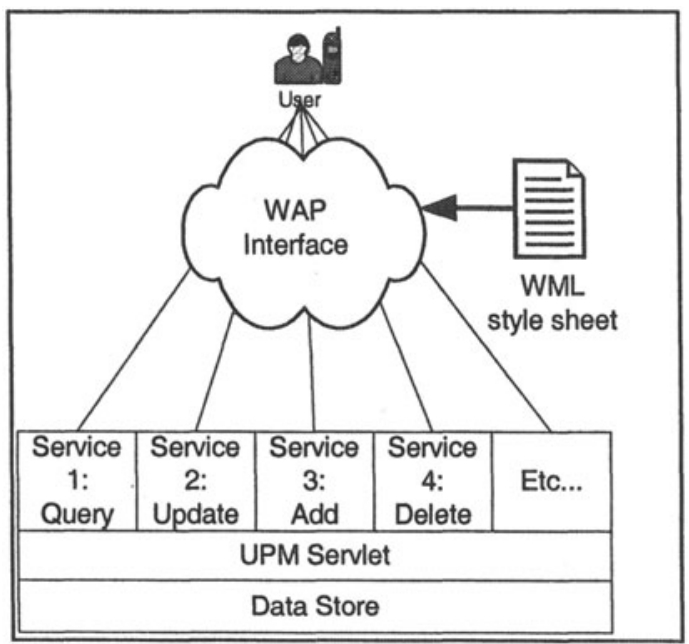

Figure 3. User updating the User Profile through a dynamically created user interface

The user must be able to access his/her profile to read and modify settings for existing terminals and add new terminals. This should be done through any kind of terminal, e.g. Web browser, mobile phone, PDA, etc. This means that functionality for reading from and updating the database information must be exposed to the user and a suitable user interface must be provided.

The UPM can apply XSLT style sheets to XML documents in order to transform the documents into HTML (XHTML) or WML (or any other desired mark up) according to what type of device is calling the service. See [6][7] for more details on possible architecture and implementation. Figure 3 shows a user accessing the user profile through a WAP interface.

\subsection{3rd party application access to user profile data and functions}

The end user application provided by any 3rd party must get access to the UPWS in order to configure according to the settings defined in the profile. The application should not have access to the whole profile, merely the parts relevant for the type of terminal and service in use, and in according to user 
specifications. Conforming to de facto Web service standards, the UPWS uses the SOAP protocol for wrapping data to be exchanged between the user profile and the application. Based on the information retrieved from the profile service, the application can provide the user with a personalised service, as shown in Figure 4.

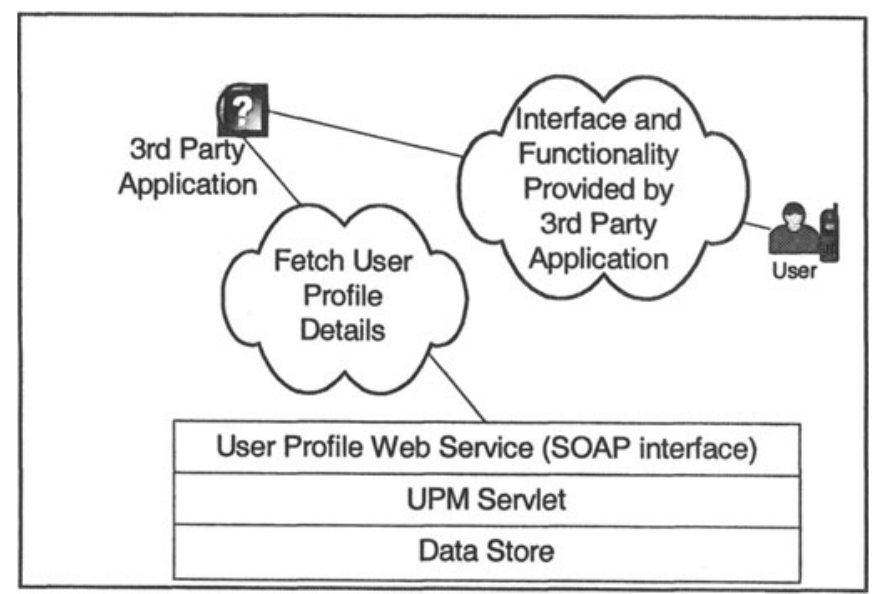

Figure 4. Application using the UPWS for personalisation of its services to the end user

It might be desirable that the user be able to access the profile through the 3rd party application. The functionality required is the same as if the user accessed the profile directly, but the UPM does not need to provide any user interface, since that will be taken care of by the 3rd party application. However, a SOAP interface must be provided so that the 3rd party application can interact with the services.

\subsection{Use Scenarios}

1. The user accesses the profile through a mobile phone. After the user logs in through the UPM, the UPM extracts the correct user profile data from the database and wraps them in XML. Since the terminal is a WML browser, a WML style sheet is used to create WML pages that displays the desired parts of the XML document that constitutes the user's profile. Through the UPM the user gets access to the data and can update the User Profile. All needed functionality is disclosed to the user through the WAP interface.

2. The user uses an application that utilises the UPWS. In order to provide a personalised service to the user, the $3^{\text {rd }}$ party application contacts the UPWS and fetches the nessesary user data. These are then used to configure the interaction with the user. The user never 
has to worry about the profile, since all communication with the UPWS is between the UPWS and the $3^{\text {rd }}$ party application.

3. The user modifies his/her User Profile while using a $3^{\text {rd }}$ party application. In this scenario the application must be able to update the database, using the same functions as in scenario 1, but this time the interface is not WML, but SOAP. The user performs the same functions, but through the user interface provided by the application, instead of the interface provided by the UPM.

\section{CONCLUSION}

We have suggested that it can be useful for telecom operators to offer User Profiles as XML Web services in order to provide $3^{\text {rd }}$ party applications access to user data, preferences and settings. This way the application providers can offer customised services to the user anytime, anywhere and on any device, without the user having to maintain the same data in several applications.

Our work is in an early stage, and further work must be done in order to decide on implementation details. Choice of platform (J2EE based or .NET) is one of the significant decisions to be made. When considering platforms and other implementation details, interoperability issues inevitably become an important aspect, as does issues concerning security and reliability.

\section{REFERENCES}

[1] IBM Web Services Architecture Team. Web Services Architecture Overview. [Online]. Accessible: http://www-106.ibm.com/developerworks/library/w-ovr/, 2000 [2001, 18. December]

[2] Hagel III, J., \& Brown, J. S. Your Next IT Strategy, Harward Business Review Vol. 79, Issue 9, 2001 p.105-113.

[3] ITU-TS Universal Personal Telecommunication (UPT) Service Description, version 10 editio, January 1994. Draft recommendation F851, version 10

[4] TINA Consortium - Service Architecture - version 5.0 - 16 June 1997 http://www.tinac.com

[5] ETSI/3GPP - GSM- Digital cellular telecommunications system (Phase 2+) Organization of subscriber data (Release 1998) TS 03.08 V7.4.0 (2000-09)

[6] Biancheri, C., Pazzaglia, J.-C., Peddeu, G. EIHA?!? : deploying Web and WAP services using XML Technology. Sigmond Record. Vol. 30, Issue 1, 2001, p. 5-12

[7] Coyle, F.P. Breathing Life into legacy. IT Professional. Vol. 3, Issue 5, 2001 p. 17 24 